\title{
On Identification of Distribution Grids
}

\author{
Omid Ardakanian, Vincent W.S. Wong, Roel Dobbe, Steven H. Low, Alexandra von Meier, Claire Tomlin, Ye Yuan
}

\begin{abstract}
Large-scale integration of distributed energy resources into residential distribution feeders necessitates careful control of their operation through power flow analysis. While the knowledge of the distribution system model is crucial for this type of analysis, it is often unavailable or outdated. The recent introduction of synchrophasor technology in low-voltage distribution grids has created an unprecedented opportunity to learn this model from high-precision, time-synchronized measurements of voltage and current phasors at various locations. This paper focuses on joint estimation of model parameters (admittance values) and operational structure of a poly-phase distribution network from the available telemetry data via the lasso, a method for regression shrinkage and selection. We propose tractable convex programs capable of tackling the low rank structure of the distribution system and develop an online algorithm for early detection and localization of critical events that induce a change in the admittance matrix. The efficacy of these techniques is corroborated through power flow studies on four three-phase radial distribution systems serving real household demands.
\end{abstract}

Index Terms-System identification, event detection, phasor measurement units, distribution grids, smart grids.

\section{INTRODUCTION}

D ISTRIBUTION grids are traditionally sized in a way that they will not be stressed even under severe loading conditions. The emergence of demand side technologies and distributed energy resources (DER), such as solar panels, wind turbines, battery storage systems, and plug-in electric vehicles, has led to an unprecedented amount of variability across multiple timescales which cannot be simply managed using the traditional "fit and forget" philosophy. This manifests the need for a novel operation paradigm which is centered around pervasive monitoring, real-time analytics and control at the distribution scale [1].

In recent years, numerous smart grid technologies have been piloted to improve observability and controllability of distribution grids, examples of which are distribution supervisory control and data acquisition (D-SCADA) system and synchrophasor technology [2] - inexpensive, high-fidelity micro-phasor measurement units ( $\mu$ PMUs) sampling voltage and current waveforms at high frequency [3]. The availability of telemetry data from multiple points across a distribution network makes valuable new applications possible, such as event detection and classification, model validation, distributed generation (DG) characterization, state estimation, equipment

Omid Ardakanian is with the Department of Computing Science, University of Alberta, Canada. Vincent W.S. Wong is with the Department of Electrical and Computer Engineering, University of British Columbia, Canada Steven H. Low is with the Department of Computing and Mathematical Science, California Institute of Technology, USA. Roel Dobbe, Alexandra von Meier, and Claire Tomlin are with the Department of Electrical Engineering and Computer Sciences, UC Berkeley, USA. Ye Yuan is with the School of Automation, Huazhong University of Science and Technology, China. For correspondence: yye@hust.edu.cn health monitoring, and phasor-based control [4], [5]. While the knowledge of the distribution network model is crucial for most of these applications, such a model is often unavailable or outdated due to the continuous integration of distribution components and frequent reconfiguration of feeders. To address this problem, this work focuses on joint estimation of distribution system model parameters and its topology from the available telemetry data.

The distribution system identification problem has received a lot of attention lately. However, most research focuses on topology verification which entails identifying the subset of distribution lines that are energized using smart meter data or phasor measurements, and little effort has been put into learning the impedance parameters of lines and transformers from the available data 1 . For example, the correlation between node voltage measurements is leveraged in [6] to detect the grid topology via a sparse Markov random field. A data-driven online algorithm is proposed in [7] for detecting a switching event by comparing a trend vector built from $\mu$ PMU data with a given library of signatures derived from the possible topology changes. In [8], the optimal placement of sensors in a distribution network is investigated in order to infer the status of switches from the measurements using the maximum likelihood method. A mutual information-based algorithm is proposed in [9] to identify the distribution topology by building a graphical model that describes the probabilistic relationship among voltage measurements. In [10], a graphical model learning algorithm is proposed based on conditional independence tests for nodal voltage measurements. Principal component analysis is employed in [11] to obtain a lower dimensional subspace of the available $\mu \mathrm{PMU}$ data and project the original data onto this subspace by learning coefficients of the basis matrix using an adaptive training method. An online event detection algorithm is then proposed to approximate phasor measurements using these coefficients, issuing an alert whenever a significant approximation error is noticed.

The inverse power flow (iPF) problem, originally defined in [12], concerns recovering the admittance matrix of a power system from a sequence of voltage and current phasor measurements corresponding to different steady states of the system. In this paper, we study the iPF problem in the context of a poly-phase distribution system (loopy or radial) where each node is equipped with a sensor (i.e., the full observability assumption). Drawing on sparsity-based regularization techniques [13]-[15], we present a tractable convex program to uniquely identify the admittance matrix of the distribution system when the identification problem is well-posed. To tackle the low rank structure of a distribution network, we

\footnotetext{
${ }^{1}$ Due to the imprecise and possibly correlated phasor measurements, model parameter estimation will be nontrivial even if the true topology is known.
} 
develop a novel algorithm based on matrix decomposition which is capable of identifying a large submatrix of the admittance matrix. Furthermore, we put forward an online algorithm for early detection and localization of critical events that change the admittance matrix.

This paper extends our prior work [16] in three main ways. First, we propose the use of the adaptive lasso penalty to estimate large elements of the admittance matrix, attenuating the bias problem. Second, we propose a regularization technique that leverages the approximate knowledge of the admittance matrix to enhance identification. Third, we validate the efficacy of the proposed algorithms and study their sensitivity to the measurement error through extensive simulations on IEEE 13, 34, 37, and 123-bus feeders serving real and synthetic loads.

The closest lines of work to ours are [17], [18] which jointly address topology detection and model parameter estimation problems. In [17], these problems are merely studied in a radial network setting and the results are not extended to poly-phase and mesh systems. In [18], noisy measurements of power injections and voltage phasors from $\mu$ PMUs and smart meters are leveraged for the joint estimation of line parameters and topology of a distribution system. However, their approach cannot be used to detect faults or track topology changes.

\section{Problem Formulation}

This section describes the iPF problem in a poly-phase distribution system and introduces a regularization technique for simultaneous estimation and variable selection to efficiently solve this problem under certain assumptions.

\section{A. Preliminaries}

We denote the set of complex matrices and the set of symmetric complex matrices by $\mathbb{C}$ and $\mathbb{S}$, respectively, the transpose of a matrix $A$ by $A^{\top}$, its Hermitian (complex conjugate) transpose by $A^{H}$, its pseudo-inverse by $A^{\dagger}$, its Frobenius norm by $\|A\|_{F}$, and the smallest number of linearly dependent columns of this matrix by $\operatorname{Spark}(A)$. All-zeros and all-ones vectors are denoted respectively by $\mathbf{0}$ and $\mathbf{1}$, and the cardinality of a set $\mathcal{N}$ is denoted by $|\mathcal{N}|$. Placing a caret over a letter indicates that it represents an estimated value.

A poly-phase power distribution system can be modeled by an undirected graph $\mathcal{G}=(\mathcal{N}, \mathcal{E})$ where $\mathcal{N}=\{1,2, \ldots, N\}$ represents the set of nodes, and $\mathcal{E} \subseteq \mathcal{N} \times \mathcal{N}$ represents the set of energized lines, each connecting two distinct nodes. We denote the phases of a node $n \in \mathcal{N}$ by $\mathcal{P}_{n} \subseteq\left\{a_{n}, b_{n}, c_{n}\right\}$ and the phases of a line $(m, n) \in \mathcal{E}$ connecting node $m$ to node $n$ by $\mathcal{P}_{m n} \subseteq\left\{a_{m n}, b_{m n}, c_{m n}\right\}$. For node $n \in \mathcal{N}$ and phase $\phi \in \mathcal{P}_{n}$, let $V_{n}^{\phi} \in \mathbb{C}$ be its line-to-ground voltage and $I_{n}^{\phi} \in \mathbb{C}$ be the injected current. We represent the voltages and injected currents of different phases of node $n \in \mathcal{N}$ by vectors $V_{n}=\left\{V_{n}^{\phi}\right\}_{\phi \in \mathcal{P}_{n}}$ and $I_{n}=\left\{I_{n}^{\phi}\right\}_{\phi \in \mathcal{P}_{n}}$, respectively, and use the per-unit system to express the quantities. We treat the voltage at the distribution substation as reference for phasor representation.

We model lines as $\pi$-equivalent components and denote the phase impedance and shunt admittance matrices of line $(m, n)$ by $Z_{m n} \in \mathbb{C}^{\left|\mathcal{P}_{m n}\right| \times\left|\mathcal{P}_{m n}\right|}$ and $Y_{m n}^{s} \in \mathbb{C}^{\left|\mathcal{P}_{m n}\right| \times\left|\mathcal{P}_{m n}\right|}$, respectively. Similarly, transformers are modeled as series components with an admittance matrix which depends on the type of connection. Assembling the admittance matrices of all components, the admittance matrix can be constructed for the distribution system, denoted by $Y_{\text {bus }} \in \mathbb{S}^{\sum_{n \in \mathcal{N}}\left|\mathcal{P}_{n}\right| \times \sum_{n \in \mathcal{N}}\left|\mathcal{P}_{n}\right|}$, which is a symmetric matrix that satisfies $Y_{\text {bus }} \mathbf{1}=\mathbf{0}$ if shunt elements are neglected. The bus admittance matrix relates the node voltages and injected currents according to Ohm's law:

$$
\underbrace{\left[\begin{array}{c}
I_{1}(k) \\
I_{2}(k) \\
\vdots \\
I_{N}(k)
\end{array}\right]}_{I_{\text {bus }}(k)}=\underbrace{\left[\begin{array}{cccc}
Y_{11} & Y_{12} & \ldots & Y_{1 N} \\
Y_{12}^{\top} & Y_{22} & \ldots & Y_{2 N} \\
\vdots & \vdots & \ddots & \vdots \\
Y_{1 N}^{\top} & Y_{2 N}^{\top} & \ldots & Y_{N N}
\end{array}\right]}_{Y_{\text {bus }}} \underbrace{\left[\begin{array}{c}
V_{1}(k) \\
V_{2}(k) \\
\vdots \\
V_{N}(k)
\end{array}\right]}_{V_{\text {bus }}(k)},
$$

where $k=1, \ldots, K$ is the time index, $V_{\text {bus }}(k), I_{\text {bus }}(k) \in$ $\mathbb{C}^{\sum_{n \in \mathcal{N}}\left|\mathcal{P}_{n}\right| \times 1}$ are steady-state complex nodal voltages and injected currents at time $k$, each off-diagonal block of $Y_{\text {bus }}$ is a submatrix $Y_{m n}=-Z_{m n}^{-1}$ corresponding to the admittance of line $(m, n)$, and each diagonal block is a submatrix

$$
Y_{n n}=\sum_{m \in\{o \mid(o, n) \in \mathcal{E}\}}\left(\frac{1}{2} Y_{m n}^{s}+Z_{m n}^{-1}\right) .
$$

Rewriting (1) in vector form for $K$ time slots yields:

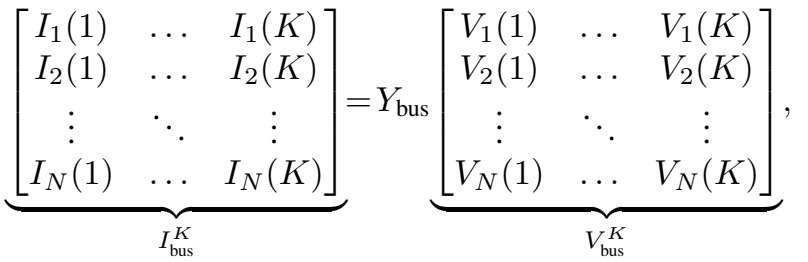

where $V_{\text {bus }}^{K}$ and $I_{\text {bus }}^{K}$ collect nodal voltages and injected currents sampled at $K$ successive time slots, respectively.

The iPF problem that we study in this paper concerns recovering the admittance matrix of a poly-phase distribution system, $Y_{\text {bus }}$, from voltage and current phasor measurements of all nodes, $V_{\text {bus }}^{K}$ and $I_{\text {bus }}^{K}$. In general, $V_{\text {bus }}^{K}$ is low rank in a power system ${ }^{2}$, making the identification problem ill-posed. In the following, we first study how the admittance matrix can be identified when the identification problem is well-posed. We discuss in Section III how a large part of the admittance matrix can be identified despite the low rank structure of $V_{\text {bus }}^{K}$.

\section{B. Sparsity-based Regularization}

This section presents a robust algorithm for recovering the admittance matrix of a distribution system from noisy sensor dat 3 under two assumptions: (a) the identification problem is well-posed, and (b) all nodes can be monitored.

\footnotetext{
${ }^{2}$ This is reported for transmission PMU data in $[11$ and is further supported by the experiments on real $\mu \mathrm{PMU}$ data obtained from [19].

${ }^{3}$ Existing synchrophasor technology is capable of sampling voltage and current waveforms at $120 \mathrm{~Hz}$ or higher [3]. But even at a lower temporal resolution (e.g., one-minute), enough data can be collected to identify the model of a distribution network comprised of several hundred nodes, before it changes. Nevertheless, for convenience, we henceforth refer to the available sensor data as $\mu \mathrm{PMU}$ data.
} 
The admittance matrix can be identified by solving the following regression problem:

$$
\begin{aligned}
\widehat{Y}_{\text {bus }}= & \underset{Y}{\operatorname{argmin}}\left\|Y V_{\text {bus }}^{K}-I_{\text {bus }}^{K}\right\|_{F} \\
& \text { subject to } \quad Y \in \mathbb{S}^{N \times N} .
\end{aligned}
$$

In practice, the sample size $K$ can be smaller than the number of unknown variables in the admittance matrix and we seek for a sparse solution because $Y_{\text {bus }}$ encodes the topology of a distribution network $4^{4}$. Thus, we adopt sparsity-based regularization techniques to identify the admittance matrix. We specifically enforce sparsity of $Y_{\text {bus }}$ by applying the vec operator, which converts a matrix into a column vector, to the objective function and constraining the $\ell_{0}$-norm of $\operatorname{vec}\left(Y_{\text {bus }}\right)$ :

$$
\begin{array}{r}
\hat{Y}_{\text {bus }}=\underset{Y}{\operatorname{argmin}}\left\|\left(\left(V_{\text {bus }}^{K}\right)^{\top} \otimes \mathbb{1}_{N}\right) \operatorname{vec}(Y)-\operatorname{vec}\left(I_{\text {bus }}^{K}\right)\right\|_{2} \\
\text { subject to } \quad Y \in \mathbb{S}^{N \times N}, \quad\|\operatorname{vec}(Y)\|_{0} \leq \delta,
\end{array}
$$

where $\otimes$ is the Kronecker product and $\delta$ determines the degree of sparsity of $Y_{\text {bus. }}$. The cardinality constraint makes this problem NP-hard [20].

Exploiting the symmetric structure of $Y_{\text {bus }}$, we reduce the number of parameters that need to be estimated. Consider a mapping $f: \mathbb{C}^{N \times N} \rightarrow \mathbb{C}^{\left(N^{2}+N\right) / 2 \times 1}$ which collects the lower triangular elements of a complex matrix as illustrated below:

$$
f(A)=\left[a_{11}, a_{21}, a_{31}, \ldots a_{N 1}, a_{22}, a_{32}, \ldots a_{N 2}, \ldots a_{N N}\right]^{\top},
$$

where $a_{i j}$ is the element in the $i$ th row and $j$ th column of matrix $A$. Observe that $f$ is a bijection for any $Y \in \mathbb{S}^{N \times N}$ and we have $\operatorname{vec}(Y)=Q_{Y} f(Y)$, where $Q_{Y} \in \mathbb{R}^{N^{2} \times\left(N^{2}+N\right) / 2}$ is a unique binary matrix that converts $f(Y)$ to $\operatorname{vec}(Y)$. Hence, the iPF problem can be reformulated as:

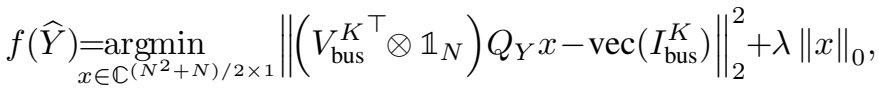
where $\lambda$ is a suitable positive regularization parameter. The above problem is non-convex and cannot be solved efficiently; thus, we solve a convex relaxation of this problem known as the lasso [21], hoping that the solutions coincide 5 The penalized form of lasso can be written as:

$$
\min _{x \in \mathbb{C}^{\left(N^{2}+N\right) / 2 \times 1} \|}\|\underbrace{\left(V_{\text {bus }}^{\left.K^{\top} \otimes \mathbb{1}_{N}\right) Q_{Y}} x\right.}_{A}-\underbrace{\operatorname{vec}\left(I_{\text {bus }}^{K}\right)}_{b}\|_{2}^{2}+\lambda\|x\|_{1} .
$$

The lasso continuously shrinks the elements of $Y_{\text {bus }}$ toward 0 as $\lambda$ increases, and some coefficients are shrunk to exact 0 if $\lambda$ is sufficiently large. Hence, selecting $\lambda$ is critical to the performance of the lasso and cross-validation can be used for this purpose. We note that (5) can be solved using a standard convex optimizer as well as iterative algorithms [22]-[24], which are more compelling in large distribution networks.

\footnotetext{
${ }^{4}$ Distribution systems typically have a radial operational structure since they are operated in such a way that power flows on a radial sub-graph at any particular time. Hence, most elements of the admittance matrix are zero.

${ }^{5}$ In [14], conditions are established for the solution of $\ell_{1}$ optimization to coincide with the solution of $\ell_{0}$ optimization.
}

Once $f(\widehat{Y})$ is recovered, $\operatorname{vec}(\widehat{Y})$ can be easily constructed:

$$
\operatorname{vec}(\widehat{Y})=Q_{Y} f(\widehat{Y}) .
$$

It is shown in [12] that the proposed technique can solve the identification problem when it is well-posed.

\section{Avoiding Unnecessary Bias}

Despite significant statistical and computational advantages of the lasso for solving the iPF problem, it is not an 'oracle procedure' and could result in suboptimal estimation in certain cases [25]. The lasso equally penalizes all elements of $Y_{\text {bus }}$, thereby producing biased estimates for the large elements. Hence, it may fail to identify the true admittance matrix when the distribution system contains several switches and voltage regulators which have much larger admittance than the distribution lines.

To avoid the unnecessary bias, we can assign data-dependent weights to different elements of $Y_{\text {bus }}$ in the $\ell_{1}$ penalty. The two-stage algorithm, known as the adaptive lasso [25], applies less shrinkage whenever the true unknown variable is large. Specifically, $f(Y)$ can be recovered from:

$$
\min _{x \in \mathbb{C}^{\left(N^{2}+N\right) / 2 \times 1}}\|A x-b\|_{2}^{2}+\lambda \sum_{i} \frac{\left|x_{i}\right|}{\left|\hat{x}_{i}\right|^{\gamma}},
$$

where $\gamma$ is a positive parameter and $\hat{x}_{i}$ is an initial estimator for $x_{i}$, e.g., the ordinary least squares (OLS) estimator defined:

$$
\hat{x}=\left(A^{\top} A\right)^{-1} A^{\top} b .
$$

Rescaling the columns of $A=\left(V_{\text {bus }}^{K^{\top}} \otimes \mathbb{1}_{N}\right) Q_{Y}$ with the corresponding weights, i.e., $\left|\hat{x}_{i}\right|^{\gamma}$, reduces (7) to the standard lasso problem, and therefore, it can be solved using the same algorithms developed for the lasso. Note that two-dimensional cross-validation is typically used to tune $(\lambda, \gamma)$.

In Section $\mathrm{V}$, we compare the lasso and the adaptive lasso penalties and show that the adaptive lasso outperforms the lasso in terms of identification accuracy in the test feeders.

\section{Exploiting Additional Structure}

Distribution circuits are upgraded and reconfigured to meet the growing demand of a neighbourhood, accommodate new technologies installed at customers' premises, and minimize losses. These changes are seldom incorporated into the distribution system model; thus, the available model is usually obsolete and cannot be relied on for diagnostics and control applications. We now discuss how such an approximate model can be leveraged to improve the identification accuracy.

We represent the available (and presumably inaccurate) admittance matrix of a distribution system by $\widetilde{Y} \triangleq Y_{\text {bus }}+\Psi$ where $Y_{\text {bus }}$ is the true admittance matrix of the network, which we ultimately intend to find, and $\Psi$ is an arbitrary error matrix that must be identified. We note that $\Psi$ is symmetric since both $\widetilde{Y}$ and $Y_{\text {bus }}$ are symmetric. If all elements of $\Psi$ are small 6

\footnotetext{
${ }^{6}$ Alternately, $\Psi$ might have a small number of nonzero elements that are not necessarily small, e.g., when the only unknown information is the status of switches which typically have large admittance values. In such cases, the Frobenius norm of $\Psi$ in 8 must be replaced with the $\ell_{0}$-norm of $\Psi$.
} 
the identification problem reduces to solving the following regularized least squares problem:

$$
\widehat{Y}_{\text {bus }}=\tilde{Y}-\underset{\Psi \in \mathbb{S}^{N} \times N}{\operatorname{argmin}}\left\|(\tilde{Y}-\Psi) V_{\text {bus }}^{K}-I_{\text {bus }}^{K}\right\|_{F}^{2}+\lambda\|\Psi\|_{F}^{2},
$$

where $\lambda$ is a tuning parameter.

Exploiting the symmetric structure of $\Psi$ and adopting the technique outlined in Section II-B, we can solve the following ridge regression problem to identify $\Psi$ :

$$
f(\widehat{\Psi})=\underset{x \in \mathbb{C}\left(N^{2}+N\right) \times 1}{\operatorname{argmin}}\|A x-b\|_{2}^{2}+\lambda\|x\|_{2}^{2}
$$

where $A=-\left(V_{\text {bus }}^{K^{\top}} \otimes \mathbb{1}_{N}\right) Q_{\Psi}$ and $b=\operatorname{vec}\left(I_{\text {bus }}^{K}-\tilde{Y} V_{\text {bus }}^{K}\right)$. This approach can be used to periodically update the distribution system model.

\section{Low RAnk Structure of Distribution Systems}

The voltage measurement matrix, $V_{\text {bus }}^{K}$, is low rank in most power distribution systems due to the interdependencies between nodal voltages. This results in an ill-posed problem that cannot be solved to identify the admittance matrix in its entirety even if $K \gg N$. To tackle this problem, we propose a novel identification algorithm based on a particular partitioning of $V_{\text {bus }}^{K}$ into two matrices, one of which has full row rank; this permits us to recover at least some part of the admittance matrix while the rest of it cannot be recovered. The steps of this algorithm are described below.

\section{A. Similarity Transformation}

Let $R$ be the row rank of $V_{\text {bus }}^{K}$. We partition $V_{\text {bus }}^{K}$ into two matrices via a similarity transformation of the matrix $Y_{\text {bus }}$ :

$$
\underbrace{\mathcal{T} I_{\text {bus }}^{K}}_{\mathbb{V}}=\underbrace{\left(\mathcal{T} Y_{\text {bus }} \mathcal{T}^{-1}\right)}_{\mathbb{V}}(\underbrace{\mathcal{T} V_{\text {bus }}^{K}}_{\mathbb{V}})
$$

where $\mathcal{T}$ is a $\sum_{n \in \mathcal{N}}\left|\mathcal{P}_{n}\right| \times \sum_{n \in \mathcal{N}}\left|\mathcal{P}_{n}\right|$ matrix that splits $V_{\text {bus }}^{K}$ into an $R \times K$ matrix, denoted by $\bigvee_{2}$, containing $R$ linearly independent rows of $V_{\text {bus }}^{K}$ and an $\left(\sum_{n \in \mathcal{N}}\left|\mathcal{P}_{n}\right|-R\right) \times K$ matrix, denoted by $\mathbb{V}_{1}$, containing other rows of $V_{\text {bus }}^{K}$ that are all in the row space of $\bigvee_{2}$. Algorithm 1 describes the steps for building these two submatrices from the available synchrophasor data. Shuffling rows of $V_{\text {bus }}^{K}$ and $I_{\text {bus }}^{K}$ according to this transformation yields:

$$
\mathcal{T} V_{\text {bus }}^{K}=\left[\begin{array}{l}
\mathbb{V}_{1} \\
\mathbb{V}_{2}
\end{array}\right], \quad \mathcal{T} I_{\text {bus }}^{K}=\left[\begin{array}{l}
\square_{1} \\
\square_{2}
\end{array}\right] .
$$

Since $\mathbb{V}_{1}$ is in the row space of $\mathbb{V}_{2}$, we can estimate the basis $X$ such that $\mathbb{V}_{1}=X \bigvee_{2}$ from $\mu$ PMU data by computing the pseudo-inverse of $\mathbb{V}_{2}: X=\mathbb{V}_{1} \mathbb{V}_{2}^{\dagger}$. Note that the pseudoinverse is well-defined here since $\mathbb{V}_{2}$ is full row rank.

\section{B. Recovering Parts of $Y_{\text {bus }}$}

We write 10 as

$$
\left[\begin{array}{l}
\mathfrak{q}_{1} \\
\mathbb{\square}_{2}
\end{array}\right]=\underbrace{\left[\begin{array}{ll}
\mathbb{Y}_{1,1} & \mathbb{Y}_{1,2} \\
\mathbb{Y}_{1,2}^{\top} & \mathbb{Y}_{2,2}
\end{array}\right]}_{\mathbb{Y}}\left[\begin{array}{c}
X \mathbb{V}_{2} \\
\mathbb{V}_{2}
\end{array}\right]=\left[\begin{array}{c}
\mathbb{Y}_{1,1} X+\mathbb{Y}_{1,2} \\
\mathbb{Y}_{1,2}^{\top} X+\mathbb{Y}_{2,2}
\end{array}\right] \mathbb{V}_{2},
$$

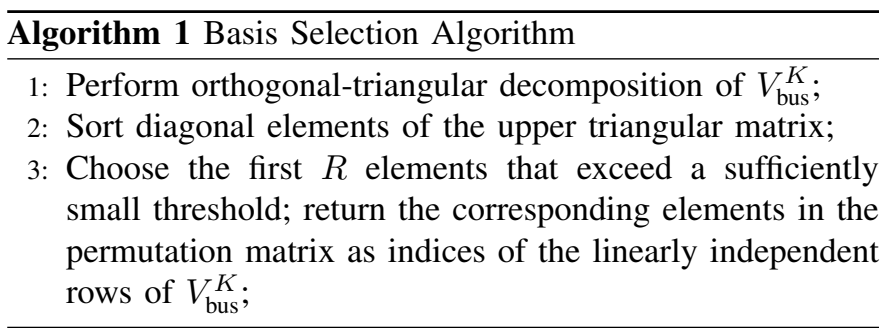

where $\left\{\Psi_{i, j}\right\}_{i, j \in\{1,2\}}$ are four submatrices of $\mathbb{Y}$ obtained according to the decomposition of $\mathbb{V}$. Note that $\mathbb{V}_{2}$ has full row rank. We have

$$
\begin{aligned}
& \mathbb{\square}_{1}=\left(\mathbb{Y}_{1,1} X+\mathbb{Y}_{1,2}\right) \mathbb{V}_{2}, \\
& \mathbb{\square}_{2}=\left(\mathbb{Y}_{1,2}^{\top} X+\mathbb{Y}_{2,2}\right) \mathbb{V}_{2} .
\end{aligned}
$$

Solving (12) for $\Psi_{1,2}$ and substituting it into (13) yields:

$$
-X^{\top} \Upsilon_{1,1} X+\mathbb{Y}_{2,2}=C,
$$

in which $C=\mathbb{q}_{2} \mathbb{V}_{2}^{\dagger}-\left(\mathbb{V}_{2}^{\dagger}\right)^{\top} \mathbb{q}_{1}^{\top} X$ can be computed from the $\mu \mathrm{PMU}$ data. Vectorizing both sides of the equation yields:

$$
-\left(X^{\top} \otimes X^{\top}\right) \operatorname{vec}\left(\mathbb{Y}_{1,1}\right)+\operatorname{vec}\left(\mathbb{Y}_{2,2}\right)=\operatorname{vec}(C) .
$$

This problem can be written in the following form to reduce the number of parameters that need to be estimated using bijection $f$ and matrix $Q$ :

$$
-\left(X^{\top} \otimes X^{\top}\right) Q_{Y_{11}} f\left(\mathbb{Y}_{1,1}\right)+Q_{Y_{22}} f\left(\mathbb{Y}_{2,2}\right)=\operatorname{vec}(C) .
$$

Enforcing sparsity of the components of $Y_{\text {bus }}$, it is possible to identify $\Psi_{1,1}$ and $\Psi_{2,2}$ from this optimization problem:

$$
\begin{aligned}
{\left[\begin{array}{l}
f\left(\widehat{\mathbb{Y}}_{1,1}\right) \\
f\left(\widehat{\mathbb{Y}}_{2,2}\right)
\end{array}\right]=} & \arg \min _{x} \lambda \sum_{i} w_{i}\left|x_{i}\right|+ \\
& \left\|\left[-\left(X^{\top} \otimes X^{\top}\right) Q_{Y_{11}}, Q_{Y_{22}}\right] x-\operatorname{vec}(C)\right\|_{2}^{2} .
\end{aligned}
$$

Depending on whether we use the lasso or the adaptive lasso penalty, $w_{i}$ is set to 1 or $1 /\left|\hat{x}_{i}\right|^{\gamma}$ for some $\gamma>0$.

Once this problem is solved, $\mho_{1,2}$ can be identified from 13 using the method of least squares. However, there is no guarantee that $\mho_{1,2}$ is estimated with sufficient accuracy as the error introduced in the process of estimating $\Psi_{2,2}$ propagates. We show in Section $\nabla$ that $\mathbb{Y}_{2,2}$ can be accurately estimated in all cases despite the low rank structure, while $\Psi_{1,1}$ and $\Psi_{1,2}$ cannot be recovered with sufficient accuracy.

\section{Timely Detection And Localization of Events}

Several types of power system events, such as switching actions, tap operations, arc and ground faults can change the effective admittance between the nodes, thereby resulting in a different admittance matrix. In this section, we propose an online algorithm for tracking changes in the admittance matrix of a distribution system and identifying the events that induced these changes. This algorithm requires a small amount of data and has a low false alarm rate, enabling operators to take remedial actions in quasi real-time.

Consider an affine parameterization of the admittance matrix, denoted by $Y_{\text {bus }}^{\delta(k)}$, where

$$
\delta(k)= \begin{cases}0, & k<t \\ 1, & k \geq t\end{cases}
$$


is the discrete mode and $t$ is the time that the event has occurred. Our goal is to determine $t$ and find out how the admittance matrix has changed by estimating $Y_{\text {bus }}^{1}-Y_{\text {bus }}^{0}$ using as few successive voltage and current phasor measurements as possible. The updated entries of the admittance matrix indicate the type and approximate location of the event. For instance, if two elements of the admittance matrix change in a certain way during an event, it can be attributed to a switch that was opened while another one was closed.

\section{A. Event Detection}

To detect a change in the admittance matrix, we estimate the injected current vector at time $k$ from Ohm's law using the known admittance matrix, $Y_{\text {bus }}^{0}$, and the measured voltage vector at time $k$. We then compare the estimated injected current vector $\hat{I}_{\text {bus }}$ with the measured current vector $I_{\text {bus }}$ at time $k$ to calculate the prediction error:

$$
e(k)=I_{\text {bus }}(k)-\hat{I}_{\text {bus }}(k)=I_{\text {bus }}(k)-Y_{\text {bus }}^{0} V_{\text {bus }}(k) .
$$

The series $e(\cdot)$ is white noise if the admittance matrix does not change; this can be verified by the turning point test ${ }^{7}$. When the prediction error $\|e(k)\|$ exceeds a predefined threshold, we assert that the admittance matrix has changed at time $k$.

\section{B. Event Localization}

The simplest approach to event localization is to run the identification algorithm presented in the previous section upon detection of an event to update the admittance matrix. In a large distribution system, this requires collecting and processing a considerable number of $\mu \mathrm{PMU}$ samples following the detection, implying that the identification task may not be accomplished in a timely manner. To address this shortcoming, we propose an identification algorithm that scales with the size of the network by taking advantage of the knowledge of the admittance matrix before an event and the fact that only a small number of its elements will change during the event.

Given that the effective admittance between just a small number of nodes is expected to change in an event, the difference between the two admittance matrices corresponding to the systems before and after the event must be sparse. We leverage this sparsity to recover the new admittance matrix:

$$
\begin{array}{cl}
\min _{Y_{\text {bus }}^{1}}\left\|\operatorname{vec}\left(Y_{\text {bus }}^{1}-Y_{\text {bus }}^{0}\right)\right\|_{0} & \\
\text { subject to } & I_{\text {bus }}^{t \rightarrow t+K}=Y_{\text {bus }}^{1} V_{\text {bus }}^{t \rightarrow t+K} \\
& Y_{\text {bus }}^{1} \in \mathbb{S}^{\sum_{n \in \mathcal{N}}\left|\mathcal{P}_{n}\right| \times \sum_{n \in \mathcal{N}}\left|\mathcal{P}_{n}\right|},
\end{array}
$$

in which $Y_{\text {bus }}^{0}$ is known, $t$ is the time slot when the event is detected, and

$$
I_{\text {bus }}^{t \rightarrow t+K}=\left[\begin{array}{ccc}
I_{1}(t) & \ldots & I_{1}(t+K) \\
I_{2}(t) & \ldots & I_{2}(t+K) \\
\vdots & \ddots & \vdots \\
I_{N}(t) & \ldots & I_{N}(t+K)
\end{array}\right]
$$

7 For a detailed discussion on how network topology errors can be detected, the readers can refer to [26].

$$
V_{\text {bus }}^{t \rightarrow t+K}=\left[\begin{array}{ccc}
V_{1}(t) & \ldots & V_{1}(t+K) \\
V_{2}(t) & \ldots & V_{2}(t+K) \\
\vdots & \ddots & \vdots \\
V_{N}(t) & \ldots & V_{N}(t+K)
\end{array}\right]
$$

It can be readily seen that $\Delta Y \triangleq Y_{\text {bus }}^{1}-Y_{\text {bus }}^{0}$ is a symmetric complex matrix as it is the difference of two symmetric complex matrices. Hence, we have:

$$
\begin{aligned}
& \min _{\Delta Y \in \mathbb{S}^{\sum_{n \in \mathcal{N}}\left|\mathcal{P}_{n}\right| \times \sum_{n \in \mathcal{N}}\left|\mathcal{P}_{n}\right|}}\|\operatorname{vec}(\Delta Y)\|_{0} \\
& \text { subject to } \\
& I_{\text {bus }}^{t \rightarrow t+K}-Y_{\text {bus }}^{0} V_{\text {bus }}^{t \rightarrow t+K}=\Delta Y V_{\text {bus }}^{t \rightarrow t+K},
\end{aligned}
$$

which can be relaxed and converted to the following weighted regularized $\ell_{1}$-norm optimization:

$$
\operatorname{vec}(\Delta \widehat{Y})=Q_{\Delta Y} \times \arg \min \|A x-b\|_{2}^{2}+\lambda \sum_{i} w_{i}\left|x_{i}\right|,
$$

where $A=\left(V_{\text {bus }}^{t \rightarrow t+K^{\top}} \otimes \mathbb{1}^{N}\right) Q_{\Delta Y}, b=\operatorname{vec}\left(I_{\text {bus }}^{t \rightarrow t+K}-\right.$ $\left.Y_{\text {bus }}^{0} V_{\text {bus }}^{t \rightarrow t+K}\right)$, and $w_{i}$ is the weight of the $\ell_{1}$ penalty which is defined earlier. This problem is convex and can be solved efficiently with only a small number of $\mu$ PMU samples compared to the original identification algorithm. The following proposition gives the necessary and sufficient condition for the solution of the $\ell_{0}$ minimization to converge to the true sparse difference matrix by establishing a minimum bound on the number of $\mu \mathrm{PMU}$ samples that are required.

Proposition 1 (From [13]). For any vector $z$, there exists a unique signal $w$ such that $z=\Phi w$ with $\|w\|_{0}=S$ if and only if $\operatorname{Spark}(\Phi)>2 S$.

Following this, the proposed event localization algorithm needs as many $\mu \mathrm{PMU}$ samples as required for $\operatorname{Spark}(A)$ to exceed twice the number of elements of the admittance matrix that will change during an event.

\section{Performance Evaluation}

We evaluate the efficacy of the proposed algorithms in estimating the model parameters and tracking topology changes through power flow studies on test distribution systems under various loading conditions. To carry out this evaluation, we develop a simulation framework in MATLAB which integrates built-in graphics and advanced analysis capabilities with the CVX toolbox for convex optimization [27], and the Open Distribution System Simulator (OPENDSS) [28] for power flow analysis. The OPENDSS can be controlled from MATLAB through a COM interface, allowing us to load a distribution system model, change its parameters, perform power flow calculations, and retrieve the results 8 . Figure 1 depicts the principal components of this framework.

The simulator takes as input a distribution system model, the demand profile of a certain number of homes (for a fixed number of time slots), the point of connection of each home (i.e., the node that it is connected to), and a set of events that should be simulated at specified times. These events

\footnotetext{
${ }^{8}$ The control mode is disabled in OPENDSS to ensure that the transformer taps are not automatically adjusted during a simulation. This guarantees that the admittance matrix does not change unless we trigger an event.
} 


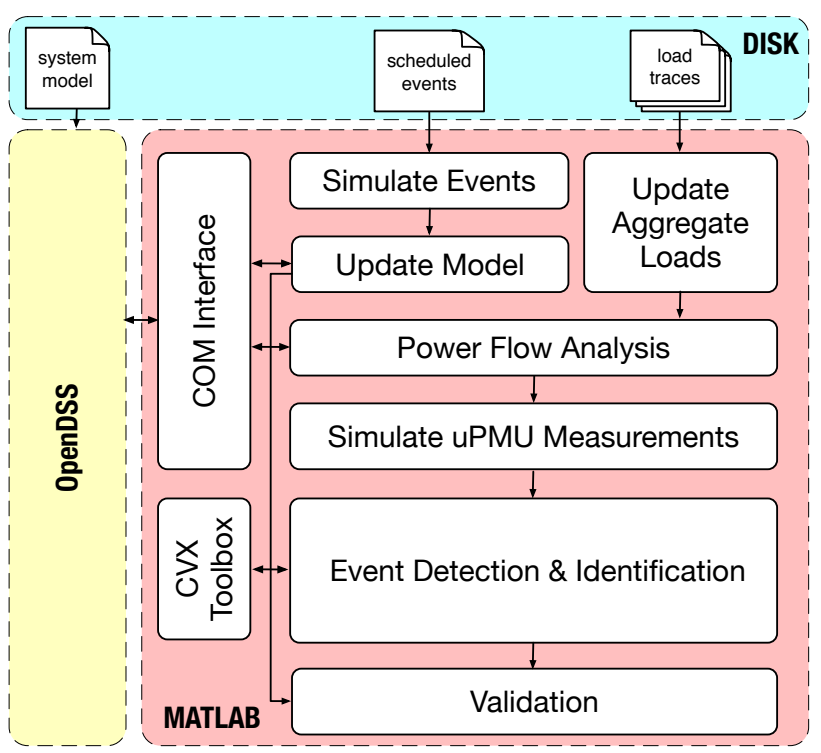

Fig. 1. A block diagram of the integrated simulation framework.

change the admittance matrix in a deterministic way and we record all these changes. Given this input data, the aggregate demand is calculated at each node and a power flow study is performed in a loop for each time slot to compute the voltage magnitude and phase angle at each node, which are treated as $\mu \mathrm{PMU}$ measurements. The proposed convex programs are then solved to recover the original admittance matrix from the available $\mu$ PMU data and update it after detecting each event. The sequence of recovered admittance matrices are eventually compared against the sequence of true admittance matrices to quantify the estimation error. We describe our test cases next.

\section{A. Distribution Feeders}

We evaluate our algorithms on four IEEE test feeders, namely 13-bus, 34-bus, 37-bus, and 123-bus systems [29]; these unbalanced radial systems operate at a nominal voltage of $4.16 \mathrm{kV}, 24.9 \mathrm{kV}, 4.8 \mathrm{kV}$, and $4.16 \mathrm{kV}$, respectively, and differ in size and sparsity as shown in Table I The columns of this table respectively represent the test feeder, the number of nodes in its OpENDSS model, the rank of $V_{\text {bus }}^{K}$ (when $K$ is much larger than the number of nodes), the percentage of $Y_{\text {bus }}$ elements that are zero (i.e., the sparsity level), and the absolute value of the largest element of the admittance matrix. The following observations can be made for each test feeder: a) $V_{\text {bus }}^{K}$ is rank deficient which implies that it is impossible to recover the full admittance matrix from the $\mu$ PMU data, b) the admittance matrix is extremely sparse, and c) the admittance matrix has at least one element that is several orders of magnitude larger than other nonzero elements, hinting at the possibility that the lasso produces biased estimates for these large elements.

Moreover, two of these test feeders contain switches which can be operated to induce a change in the admittance matrix. The 123-bus test system contains 12 switches that can be

\footnotetext{
${ }^{9}$ Such elements typically correspond to the admittance of switches and voltage regulators.
}

TABLE I

PROPERTIES OF THE RADIAL TEST FEEDERS.

\begin{tabular}{|l|c|c|c|c|}
\hline feeder & no. nodes & $\operatorname{rank}\left(V_{\text {bus }}^{K}\right)$ & sparsity level & $\left|\max \left(Y_{\text {bus }}\right)\right|$ \\
\hline 13-bus & 35 & 27 & $81.63 \%$ & $10^{7}$ \\
\hline 34-bus & 95 & 84 & $91.72 \%$ & 402.1 \\
\hline 37-bus & 117 & 109 & $92.47 \%$ & 1012.5 \\
\hline 123-bus & 275 & 254 & $97.39 \%$ & $10^{6}$ \\
\hline
\end{tabular}

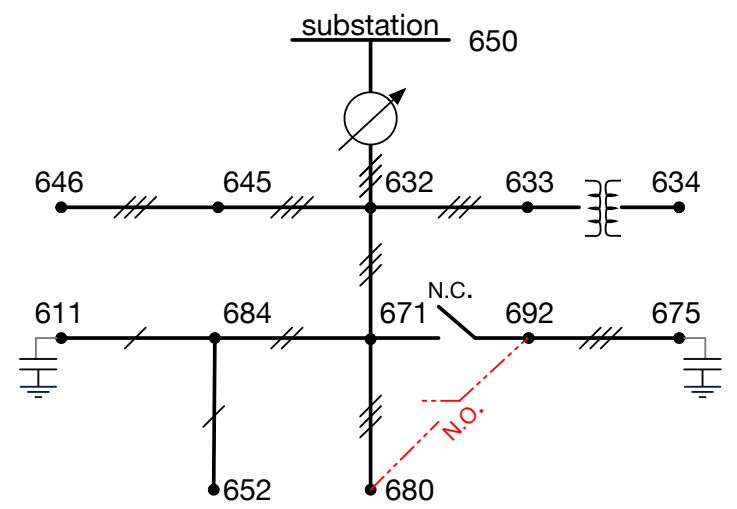

Fig. 2. A one-line diagram of the modified IEEE 13-bus test feeder. The dashed line represents the switch added to the original feeder. The number of phases connecting two nodes is shown by slashes on the lines.

operated in a certain way to change the topology while maintaining its radial structure. Hence, it provides an ideal setting for validating the event detection and localization algorithm. Similarly, the 13-bus feeder has a normally closed switch. We modify this system by adding a normally open switch with the exact same configuration between Bus 680 and Bus 692 as shown in Figure 2. This creates two feasible radial structures that span all nodes. We use this feeder to validate both event detection and system identification algorithms. The 34-bus and 37-bus feeders do not have a switch and are merely used for the purpose of validating the identification algorithm.

\section{B. Residential Loads \& Phasor Measurements}

A node in the distribution system model represents an aggregation point where usually a pole-top transformer supplies a small number of residential customers. Since distribution circuits are not modelled beyond these transformers, we aggregate demands of downstream customers at the corresponding nodes. We assume that each node is monitored by a $\mu \mathrm{PMU}$ which measures the magnitude and phase angle of the node voltage and the current drawn by the downstream customers once every time slot.

We use real data from the ADRES dataset [30] to model the customers connected to the 13-bus, 34-bus, and 37-bus test feeders. This dataset consists of high-resolution (1 second) measurements of real power, reactive power, and per-phase voltage values of 30 Austrian households over 14 days. To obtain a sufficient number of customers for our simulation, we treat a 3-phase load as three separate single-phase loads, and split 14 days of available data for each household into 14 individual loads each representing the demand of a customer over a particular day. We connect a random number of customers between 5 and 15 to each node, except the nodes 
TABLE II

ESTIMATION ERROR OF $\Upsilon_{2,2}$ BY THE LASSO AND ADAPTIVE LASSO IN DIFFERENT TEST FEEDERS.

\begin{tabular}{|c|c|c|c|c|}
\hline \multirow{2}{*}{ feeder } & \multicolumn{2}{|c|}{ lasso } & \multicolumn{2}{c|}{ adaptive lasso } \\
\cline { 2 - 5 } & M1 & M2 & M1 & M2 \\
\hline 13-bus & 4.70 & 4.71 & 1.69 & 1.77 \\
\hline 34-bus & 240.51 & 602.89 & 3.08 & 0.37 \\
\hline 37-bus & 28.70 & 65.48 & 2.71 & 2.67 \\
\hline
\end{tabular}

that are terminals of voltage regulators and switches; no load is connected to these nodes.

In the case of the IEEE 123-bus feeder, we need highresolution measurements of many households, which we lack. To address this problem, we synthesize residential loads (real power) using the continuous-time Markov models derived from fine-grained measurements of the power consumption of 20 households in Ontario [31]. We connect a random number of customers between 5 and 10 to each node, except the nodes that represent the terminals of voltage regulators and switches. Hence, the load distribution is nonuniform across different phases of a node. We consider a constant power factor of 95\% at each node, which is typical for residential loads, and set the reactive power accordingly in each time slot.

\section{Results}

To deal with the low rank structure of the test feeders, we utilize the algorithm proposed in Section III to identify the largest part of the admittance matrix that could be possibly recovered from the available data. We also utilize the event detection and localization algorithm proposed in Section IV to track how the admittance matrix changes in an event. To validate these algorithms, we perform several simulation runs for each test feeder where each simulation spans one day divided into 1-second time slots. In each case, we estimate the admittance matrix using both the lasso and the adaptive lasso penalties and compare their performance. We use two metrics to quantify the error incurred in estimating $\mathbb{\mho}_{2,2}$ :

Metric 1 (M1): $\quad\left\|\operatorname{vec}\left(\widehat{\mathbb{Y}}_{2,2}-\mathbb{Y}_{2,2}\right)\right\|_{1}$

Metric 2 (M2): $\quad\left\|\widehat{\Upsilon}_{2,2}-\widehat{\Upsilon}_{2,2}\right\|_{F}$

To tune the parameters of the convex programs, we search through a reasonable set of values. Specifically, $\gamma$ and $\lambda$ of the adaptive lasso are chosen from $\{0.5,1,2\}$ and the set of logarithmically spaced points between $10^{5}$ and $10^{-5}$, respectively. The same set of values are considered to determine $\lambda$ of the lasso. Moreover, the OLS estimator is adopted as the weight of the $\ell_{1}$ penalty in the adaptive lasso.

1) Identification with Precise Measurements: We first explore the scenario in which $\mu \mathrm{PMU}$ measurements are not affected by noise. Table $\Pi$ shows the two error metrics when the lasso or the adaptive lasso penalty is used to identify the admittance matrix of the three test feeders. In the case of the 13-bus feeder, both methods can estimate all elements of $\widehat{\mho}_{2,2}$ except one, with less than $1 \%$ relative error. The one element that is not accurately identified corresponds to the substation. Nevertheless, the adaptive lasso estimates that element with a lower error compared to the lasso as suggest by the two error metrics representing the overall accuracy of these methods. Should we leverage the knowledge of the substation type

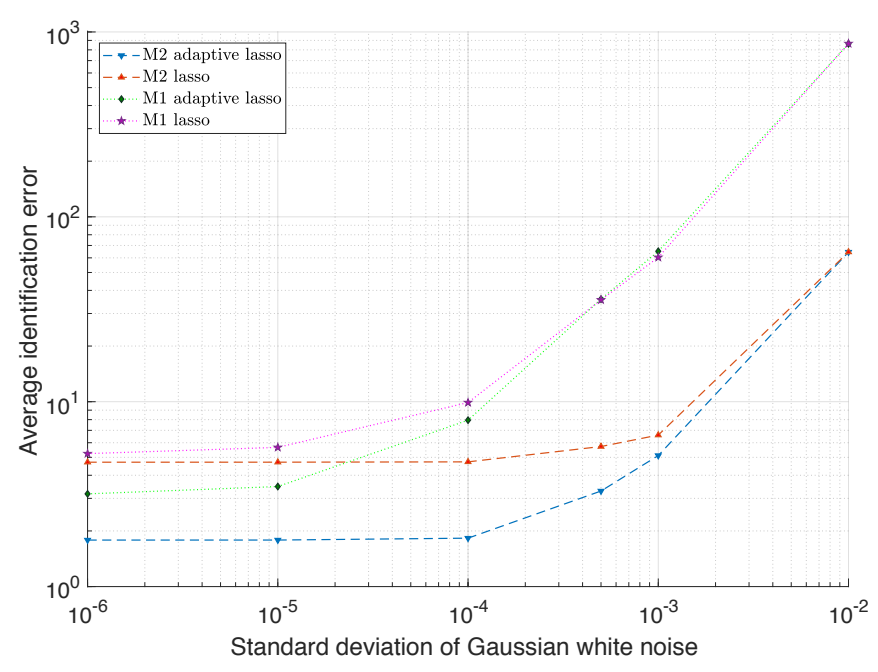

Fig. 3. Identification error of the lasso and the adaptive lasso for different noise levels in the IEEE 13-bus test feeder.

and configuration, both methods can accurately estimate all elements of $\widehat{\mathbb{Y}}_{2,2}$ (M1, M2 <0.01).

In the case of the 34-bus and 37-bus feeders, the lasso fails to accurately identify more than one element of the admittance matrix $(\mathrm{M} 2 \gg \mathrm{M} 1)$, resulting in estimation errors which are remarkably higher than the previous case. We verify that those elements have large admittance values. However, the adaptive lasso successfully estimates those elements in both feeders, resulting in relatively smaller estimation errors.

2) Identification with Noisy Measurements: We now explore the scenario in which $\mu \mathrm{PMU}$ measurements are noisy. We only show the results for the 13-bus feeder due to space limitations. To simulate the measurement error, we add a white Gaussian noise with $\sigma^{2}$ variance to node voltages and treat them as $\mu \mathrm{PMU}$ measurements $\mathrm{s}^{10}$ We try different values of $\sigma$ and perform ten simulation runs for each value. In particular, we increase the standard deviation from $10^{-6}$ to $10^{-2}$ and report the mean value of the two error metrics over these runs. Figure 3 shows the mean estimation error of the lasso and the adaptive lasso. It can be seen that both methods are quite sensitive to noise. When $\sigma=10^{-6}$, the estimation error of both methods is quite similar to the scenario with precise measurements (see Table II). The adaptive lasso can accurately identify all elements of $\widehat{\mho}_{2,2}$, except for one element (as discussed earlier) as long as $\sigma \leq 10^{-3}$. Should $\sigma$ exceeds this level, the adaptive lasso fails to identify several elements and both error metrics increase significantly. Unlike the adaptive lasso, the lasso is less robust to noise and only yields sufficiently accurate estimates when $\sigma \leq 10^{-4}$.

3) Event Detection and Localization: We finally validate the proposed event detection and localization algorithm by simulating a line tripping event and a switching operation in the 13-bus test feeder, and a switching operation in the 123bus test feeder. These events are triggered after the admittance matrix has been identified for the initial configuration of the distribution system.

\footnotetext{
${ }^{10}$ We have observed that perturbing node voltages by a small Gaussian noise does not completely eliminate the low rank problem.
} 


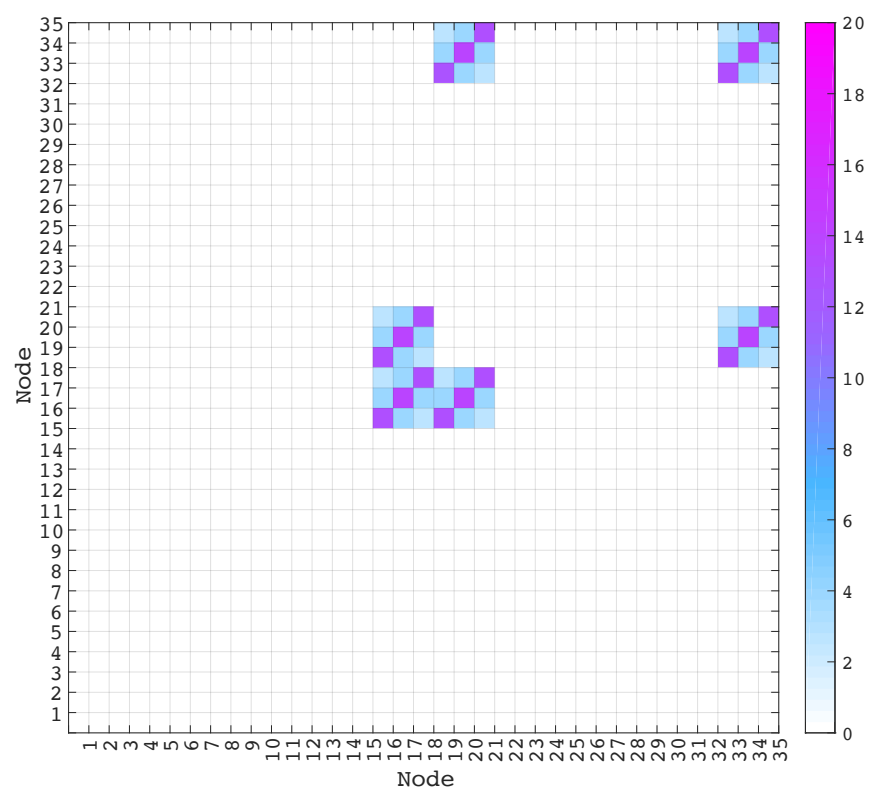

Fig. 4. A colored representation of $\Delta \widehat{Y}$ when $23 \mu$ PMU samples are used to recover the admittance matrix following the detection of the switching event.

We first focus on the 13-bus test feeder. We introduce a line tripping event by disconnecting the single-phase line between Bus 611 and Bus 684, and a switching event by closing the switch between Bus 692 and Bus 680 while opening the switch between Bus 671 and Bus 692. Both events will change the admittance matrix and therefore must be identifiable from the available data. We observe that the proposed algorithm detects the event in both cases in the same time slot that it occurs, i.e., immediately after processing the $\mu \mathrm{PMU}$ data for that time slot. For the switching event, $\Delta Y$ can be estimated with relatively high accuracy $(\mathrm{M} 1=0.17, \mathrm{M} 2=0.10)$ using $23 \mu \mathrm{PMU}$ samples following the detection of this event, as shown in Figure 4 In this figure, the color of a cell located at row $i$ and column $j$ represents the value of $\left|\widehat{Y}^{1}(i, j)-\widehat{Y}^{0}(i, j)\right|$. It can be readily seen that all elements of $\Delta \widehat{Y}$ are zero except for 6 three by three submatrices which have changed due to this event, enabling us to locate the event within a small geographical area. We verified that these submatrices correspond to the admittance of the two switches that are operated. Note that these switches are located on three-phase lines. The estimation error will increase significantly if we use fewer samples to identify $\Delta \widehat{Y}$.

Turning our attention to the 123-bus test feeder, we simulate a line switching event by closing the switch between Bus 13 and Bus 152 while opening the switch between Bus 151 and Bus 300 (refer to [32] for the topology of this feeder). The simultaneous switching operation maintains the radial structure of this distribution system. The proposed algorithm detects the event in the same time slot that it occurs and accurately identifies $\Delta \widehat{Y}(\mathrm{M} 1, \mathrm{M} 2<0.1)$ using a small number of $\mu \mathrm{PMU}$ samples following the detection.

\section{CONCLusions}

Widespread adoption of distributed energy resources in power distribution grids calls for an advanced operation paradigm centered around monitoring, diagnostics, and control. While the knowledge of the distribution system model is crucial for most diagnostics and control applications, this model is often unavailable or outdated in practice. This paper studies how the admittance matrix of a power distribution system can be identified from limited voltage and current phasor measurements of inexpensive distribution PMUs. It proposes tractable convex programs to recover the admittance matrix in various settings and to track changes in the network topology, and investigates the fundamental limitations of these techniques. There are several avenues for future work. The $\mu \mathrm{PMU}$ installation is currently limited in low-voltage distribution networks. To address this problem, we intend to develop techniques that leverage both smart meter and $\mu \mathrm{PMU}$ data to improve identifiability of low-voltage distribution grids. We also plan to develop an identification algorithm that can deal with hidden states in the network. Furthermore, the proposed technique can be computationally expensive when it is applied to a distribution network with thousands of nodes. We intend to develop a distributed identification algorithm for large distribution networks.

\section{REFERENCES}

[1] O. Ardakanian, S. Keshav, and C. Rosenberg, Integration of Renewable Generation and Elastic Loads into Distribution Grids, ser. SpringerBriefs in Electrical and Computer Engineering. Springer International Publishing, 2016.

[2] North American Synchrophasor Initiative, "Distribution Task Team," https://www.naspi.org/distt

[3] PSL, "Micro Synchrophasors," http://www.powersensorsltd.com/ PQube3.php

[4] D. B. Arnold, C. Roberts, O. Ardakanian, and E. M. Stewart, "Synchrophasor data analytics in distribution grids," in IEEE Power Energy Society Innovative Smart Grid Technologies Conference (ISGT), April 2017, pp. 1-5.

[5] A. von Meier, E. Stewart, A. McEachern, M. Andersen, and L. Mehrmanesh, "Precision Micro-Synchrophasors for Distribution Systems: A Summary of Applications," IEEE Transactions on Smart Grid, vol. 8, no. 6, pp. 2926-2936, Nov. 2017.

[6] S. Bolognani, N. Bof, D. Michelotti, R. Muraro, and L. Schenato, "Identification of power distribution network topology via voltage correlation analysis," in IEEE Decision and Control (CDC), Dec. 2013, pp. 16591664.

[7] G. Cavraro, R. Arghandeh, K. Poolla, and A. von Meier, "Data-Driven Approach for Distribution Network Topology Detection," in IEEE PES General Meeting, Jul. 2015, pp. 1-5.

[8] Y. Sharon, A. M. Annaswamy, A. L. Motto, and A. Chakraborty, "Topology identification in distribution network with limited measurements," in IEEE PES Innovative Smart Grid Technologies, Jan. 2012, pp. 1-6.

[9] Y. Weng, Y. Liao, and R. Rajagopal, "Distributed Energy Resources Topology Identification via Graphical Modeling," IEEE Transactions on Power Systems, vol. 32, no. 4, pp. 2682-2694, Jul. 2017.

[10] D. Deka, S. Backhaus, and M. Chertkov, "Estimating distribution grid topologies: A graphical learning based approach," in Power Systems Computation Conference (PSCC), Jun. 2016, pp. 1-7.

[11] L. Xie, Y. Chen, and P. R. Kumar, "Dimensionality Reduction of Synchrophasor Data for Early Event Detection: Linearized Analysis," IEEE Transactions on Power Systems, vol. 29, no. 6, pp. 2784-2794, Nov. 2014.

[12] Y. Yuan, O. Ardakanian, S. Low, and C. Tomlin, "On the inverse power flow problem," arXiv preprint:1610.06631, Oct. 2016.

[13] D. L. Donoho and M. Elad, "Optimally sparse representation in general (nonorthogonal) dictionaries via 11 minimization," Proceedings of the National Academy of Sciences, vol. 100, no. 5, pp. 2197-2202, 2003.

[14] J. A. Tropp, "Recovery of short, complex linear combinations via 11 minimization," IEEE Transactions on Information Theory, vol. 51, no. 4 , pp. 1568-1570, Apr. 2005. 
[15] V. Chandrasekaran, S. Sanghavi, P. A. Parrilo, and A. S. Willsky, "Rank-sparsity incoherence for matrix decomposition," SIAM Journal on Optimization, vol. 21, no. 2, pp. 572-596, 2011.

[16] O. Ardakanian, Y. Yuan, R. Dobbe, A. von Meier, S. Low, and C. Tomlin, "Event detection and localization in distribution grids with phasor measurement units," in IEEE PES General Meeting, 2017.

[17] D. Deka, S. Backhaus, and M. Chertkov, "Structure learning and statistical estimation in distribution networks - Part II," arXiv preprint:1502.07820, Feb. 2015.

[18] J. Yu, Y. Weng, and R. R., "PaToPa: A data-driven parameter and topology joint estimation framework in distribution grids," arXiv preprint:1705.08870, May 2017.

[19] A. von Meier, D. Culler, A. McEachern, and R. Arghandeh, "Microsynchrophasors for distribution systems," in IEEE PES Innovative Smart Grid Technologies Conference, Feb. 2014, pp. 1-5.

[20] B. K. Natarajan, "Sparse approximate solutions to linear systems," SIAM Journal on Computing, vol. 24, no. 2, pp. 227-234, Apr. 1995.

[21] R. Tibshirani, "Regression shrinkage and selection via the lasso," Journal of the Royal Statistical Society. Series B, vol. 58, pp. 267-288, 1996.

[22] I. Daubechies, M. Defrise, and C. De Mol, "An iterative thresholding algorithm for linear inverse problems with a sparsity constraint," Communications on Pure and Applied Mathematics, vol. 57, no. 11, pp. 1413-1457, 2004.

[23] B. Efron, T. Hastie, I. Johnstone, and R. Tibshirani, "Least angle regression," The Annals of Statistics, vol. 32, no. 2, pp. 407-499, Apr. 2004.

[24] J. Friedman, T. Hastie, H. Hfling, and R. Tibshirani, "Pathwise coordinate optimization," The Annals of Applied Statistics, vol. 1, no. 2, pp. 302-332, Dec. 2007.

[25] H. Zou, "The adaptive lasso and its oracle properties," Journal of the American Statistical Association, vol. 101, no. 476, pp. 1418-1429, 2006.

[26] K. A. Clements and P. W. Davis, "Detection and identification of topology errors in electric power systems," IEEE Transactions on Power Systems, vol. 3, no. 4, pp. 1748-1753, Nov. 1988.

[27] M. Grant and S. Boyd, "CVX: Matlab software for disciplined convex programming, version 2.1," http://cvxr.com/cvx Mar. 2014.

[28] EPRI, "Simulation Tool: OpenDSS," http://www.smartgrid.epri.com/ SimulationTool.aspx

[29] W. Kersting, "Radial distribution test feeders," in IEEE PES Winter Meeting, vol. 2, 2001, pp. 908-912.

[30] ADRES, "Autonomous Decentralised Renewable Energy Systems," http: //www.ea.tuwien.ac.at/projects/adres_concept/EN/

[31] O. Ardakanian, S. Keshav, and C. Rosenberg, "Markovian Models for Home Electricity Consumption," in SIGCOMM Workshop on Green Networking. ACM, 2011, pp. 31-36.

[32] Distribution Test Feeder Working Group, IEEE PES, "Distribution Test Feeders," https://ewh.ieee.org/soc/pes/dsacom/testfeeders 